\title{
Orthognathic treatment and temporomandibular disorders - part 1
}

\author{
Abstracted from \\ Al-Riyami S, Moles DR, Cunningham SJ. \\ Orthognathic treatment and temporomandibular disorders: a systematic review. \\ Part 1. A new quality-assessment technique and analysis of study characteristics and classifications. \\ Am J Orthod Dentofacial Orthop 2009; 136: 624.e1-15 \\ Address for correspondence: Salma Al-Riyami, Orthodontic Unit, UCL Eastman Dental Institute, \\ 256 Grays Inn Road, London WC1X 8LD, UK. E-mail: s.alriyami@eastman.ucl.ac.uk
}

\section{Questions: What proportion of people undergoing orthognathic treatment to correct dentofacial deformities also have temporomandibular joint disorders (TMD)?}

What proportion of orthognathic patients who do not have signs or symptoms of TMD preoperatively then develop TMD signs or symptoms postsurgery?

In individuals who have signs or symptoms of TMD preoperatively, how do these signs or symptoms change after treatment?

Data sources MEDLINE, bibliographies, and reference lists of identified publications and reviews, were utilised, along with personal communications with experts and specialists.

Study selection Randomised controlled trials (RCT), cohort studies and case-control studies were included if participants (of age 14 years or over) received orthognathic treatment. Studies were excluded if participants had either craniofacial syndromes or cleft lip or palate; a history of facial fractures from trauma; were undergoing orthognathic surgery purely to correct TMD; or orthognathic treatment and concomitant joint disc surgery; or, finally, if they were animal studies. Data extraction and synthesis Data extraction was conducted independently by two reviewers, and any discrepancies discussed until agreement was reached. A quality-assessment scale was constructed specifically for this study with sections for selection, performance, measurement and outcome, and attrition. A narrative synthesis is presented as meta-analysis was not either feasible or appropriate. Results A total of 53 articles ( 41 cohorts, 8 case-control and 3 RCT) were analysed for the review. Almost half (20) did not explicitly state whether the study was retrospective or prospective, it could be determined for the majority with 21 being retrospective, 28 prospective and, in the case, four articles not being sufficiently clear. There was great variability between studies in their assessment of any association between TMD and orthognathic treatment. This variability included how TMD was classified, the signs and symptoms recorded, and the time intervals reported. Perhaps most important was the great variation in the malocclusions in the studies. Although some studies included participants with a specific skeletal discrepancy, others included various skeletal deformities, so that comparisons were not always possible and, when carried out, could be a source of heterogeneity. Most studies that did report a reduction in TMD signs and symptoms after orthognathic treatment reported this association in skeletal Class II patients. A decrease was reported in some studies in the prevalence of signs and symptoms of more than $50 \%$ of people postsurgery, compared with the presurgery state, whereas fewer subjects with skeletal Class III or a high mandibular plane angle seemed to benefit from surgery. Thus, the participants' skeletal deformity could have had a direct impact on TMD, especially after surgery.

Conclusions The diversity of diagnostic criteria and classification methods used in the included studies makes interstudy comparisons difficult. Well-designed studies are needed that have standardised diagnostic criteria and classification methods for TMD.

\section{Commentary}

TMD, its relevance to dentistry in general, and orthodontics in particular, has been a widely and sometimes acrimoniously debated topic. $^{1-5}$ The conflict in orthodontic literature is directly related to the apparent lack of evidence on the role and contribution of occlusion as being a primary factor in TMD. The multifactorial nature of TMD with specific components related to muscle, joints and associated structures does not in any way diminish the relevance or importance to the clinical orthodontist, there is just no evidence to make occlusion the prime culprit and to that extent as a direct outcome assure patients of relief consequent to occlusal orthodontic therapy.

Most evidence suggests that orthodontic treatment can neither prevent nor cause TMD. If one looks for an association between strong skeletal disharmony and structural discrepancies in the craniofacial skeleton then some trends and clear directions emerge towards defining an enlarged envelope for orthognathic correction. A defined objective of good evidence is to indicate directions and trends that could help to redefine clinical practice, then this review traverses a large range of orthodontic literature. The conclusion, that there is a need for well-defined studies with standard criteria and classifications, is a common one in the evidence-based literature and one that has repeatedly highlighted the lack of good evidence in orthodontics. This rather diminishes the value of this review, since the authors were able to outline some vital associations between TMD and orthognathic surgery but, even more importantly, the quality assessment tool was strong enough to draw clear conclusions from hazy orthodontic literature - the major strength of the review.

The stated objectives here were explicit, to examine the association of orthognathic problems and TMD signs and symptoms, and to 
identify relevant literature that would document if interventions affected TMD symptoms. The inclusion criteria were balanced and well-defined, and the authors did well to deal with the problems, diversity and range of the published literature. A wide base of literature was incorporated, rather than deriving conclusions from a few selected RCT. The statistical analysis is descriptive and fits into the scope and content of the review. The authors defined fairly stringent outcome measures and criteria, and evaluation of the included studies was exhaustive.

The thought behind the new quality assessment scale has wider ramifications and should be complemented. All previously available tools have been directed only at epidemiological studies. It is an established fact that the orthodontic literature has few high-quality trials and studies from which conclusions may be drawn. To be able to incorporate and make inferences from cohort and case-controlled studies with specified criteria opens up a new arena in the assessment of orthodontic evidence. How bias can be eliminated, and the flow charts provided in the review, were well thought out, and could be the reference point for incorporating a much wider base of literature in controversial reviews.

The results do of course highlight the great variability between studies investigating the association of TMD and orthognathic treatment. The issue of heterogeneity also dogs the results, but some clear associations emerge. The greatest benefit in terms of reduction of TMD signs and symptoms with orthognathic surgery is in Class 2 patients, who showed a $50 \%$ improvement. It is also apparent that skeletal Class 3 and high angle cases have less than desired outcomes. The review provides some useful pointers about the association between skeletal deformity, TMD and outcome of orthognathic surgery. Evidence does not provide a clear correlation between occlusal, Skeletal and structural disharmony as the only or the primary contributory factor in TMD This does not alter the fact that an occlusal or structurally directed treatment regimen would contribute towards a favourable outcome of therapy.

The conclusions that there is a need for better designed studies with standard diagnostic criteria does not diminish the value of this review, given that it demonstrates how to encompass a larger body of literature, eliminate bias and provide strong indicators for modifying clinical directions.

\section{Anmol Kalha}

Institute of Dental Studies and Technologies, Modinagar, India

1. Macfarlane TV, Kenealy P, Kingdon HA, et al. Twenty-year cohort study of health gain from orthodontic treatment: temporomandibular disorders. Am / Orthod Dentofacial Orthop. 2009; 135: 692. e1-8.

2. Rinchuse D], Kandasamy S. Myths of orthodontic gnathology. Am J Orthod Dentofacial Orthop. 2009;136: 322-330.

3. Rinchuse DJ, Rinchuse DI, Kandasamy S. Evidence-based versus experience-based views on occlusion and TMD. Am J Orthod Dentofacial Orthop. 2005; 127: 249-254.

4. Rinchuse DJ, Rinchuse DJ. The impact of the American Dental Association's guidelines for the examination, diagnosis, and management of temporomandibular disorders on orthodontic practice. Am / Orthod. 1983; 83: 518-522.

5. Luther F. Orthodontics and the temporomandibular joint: where are we now? Part 1. Orthodontic treatment and temporomandibular disorders. Angle Orthod. 1998; 68: 295-304

Evidence-Based Dentistry (2010) 11, 82-83. doi:10.1038/sj.ebd.6400740 